\title{
ON IDEALS OF $A_{p}$ WITH BOUNDED APPROXIMATE UNITS AND CERTAIN CONDITIONAL EXPECTATIONS
}

\author{
JACQUES DELAPORTE AND ANTOINE DERIGHETTI
}

\section{Introduction}

Let $G$ be an abelian locally compact group and $H$ a closed subgroup. Hauenschild and Ludwig [11, Theorem 2.3, p. 170] obtained an explicit bijective correspondence between the set of all closed ideals of $L^{1}(H)$ and the set of all closed ideals of $L^{1}(G)$ invariant under the pointwise action of $L^{\infty}(G / H)$.

In this work, we obtain similar results for the Figà-Talamanca Herz algebra $A_{p}(G)$ of an amenable locally compact group $G$. For $1<p<\infty, A_{p}(G)$ is the Banach algebra of all functions $\sum_{n=1}^{\infty} \bar{k}_{n} * \breve{l}_{n}$ such that $\sum_{n-1}^{\infty}\left\|k_{n}\right\|_{p}\left\|l_{n}\right\|_{p^{\prime}}<\infty$ with the usual norm [12].

Given a closed normal subgroup $H$, we prove (Theorem 5) the existence of a bijection $e$ between the set of all closed ideals of $A_{p}(G / H)$ and the set of all closed ideals of $A_{p}(G)$ invariant under translations by elements of $H$. We show moreover (Corollary 12) that a closed ideal $I$ of $A_{p}(G / H)$ has a bounded approximate unit if and only if $e(I)$ has a bounded approximate unit. The converse part of this assertion is delicate: it requires, in the $L^{1}$ case (as shown by Bekka [1]), different tools of integration theory on the dual of $G$ which are missing for $G$ non-abelian and even for $G$ abelian if $p \neq 2$. We avoid this by considering $P M_{p}(G)$, the set of all $p$ pseudomeasures on $G$ and the Banach algebra $\operatorname{Hom}_{A_{p}(G)}\left(P M_{p}(G)\right)$ of all linear norm continuous maps $\Phi$ from $P M_{p}(G)$ into itself such that $\Phi(u T)=u \Phi(T)$ for $u \in A_{p}(G)$ and $T \in P M_{p}(G)$. We recall that $P M_{p}(G)$ is the ultraweak closure of the linear span of the set of right translations by elements of $G$ in the space of all bounded operators of $L^{p}(G)$; for $G$ abelian, $P M_{2}(G)$ is isomorphic to $L^{\infty}(\hat{G})$. There is a natural inclusion of $\operatorname{Hom}_{A_{p}(G / H)}\left(P M_{p}(G / H)\right)$ into $\operatorname{Hom}_{A_{p}(G)}\left(P M_{p}(G)\right)$. We prove (Theorem 10) the existence of a conditional expectation of $\operatorname{Hom}_{A_{p}(G)}\left(P M_{p}(G)\right.$ ) onto $\operatorname{Hom}_{A_{p}(G / H)}\left(P M_{p}(G / H)\right)$. This result seems to be new even for $G^{p}$ abelian and $p=2$. As an application we obtain the above mentioned result concerning the existence of approximate units in ideals of $A_{p}(G / H)$ and $A_{p}(G)$.

\section{Main definitions and notation}

We use notation and results of $[4,5]$. We recall here the most important ones. The Banach space $P M_{p}(G)$ is the norm dual of $A_{p}(G)$, the duality being given by

$$
\langle\bar{k} * \check{l}, T\rangle_{A_{p^{(G)}, P M_{p}(G)}}=\overline{\left\langle T \tau_{p} k, \tau_{p^{\prime}} l\right\rangle_{L^{p}(G), L^{p^{\prime}}{ }_{(G)}}}
$$

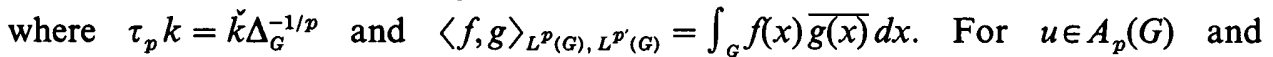
$T \in P M_{p}(G), u T$ is the operator defined by $\langle v, u T\rangle_{A_{p^{(}}(G), P M_{p}(G)}=\langle u v, T\rangle_{A_{p}(G), P M_{p^{(G)}}}$ for all $v \in A_{p}(G)$; with the mapping $(u, T) \mapsto u T, P M_{p}(G)$ is a left Banach $A_{p}(G)$-module.

Received 26 July 1991; revised 3 October 1991.

1991 Mathematics Subject Classification 43A22.

J. London Math. Soc. (2) 47 (1993) 497-506 
Let $\omega$ be the canonical map from $G$ onto $G / H$ and $\beta$ a Bruhat function for the pair $(G, H)$ (that is, $\beta$ is a non-negative continuous function on $G, \operatorname{supp} \beta \cap \omega^{-1}(K)$ is compact for every compact $K$ of $G / H$ and $\int_{H} \beta(x h) d h=1$ for every $x \in G[16$, p. 163]).

For $r, s \in C_{00}(G)$ (the space of continuous functions with compact support),

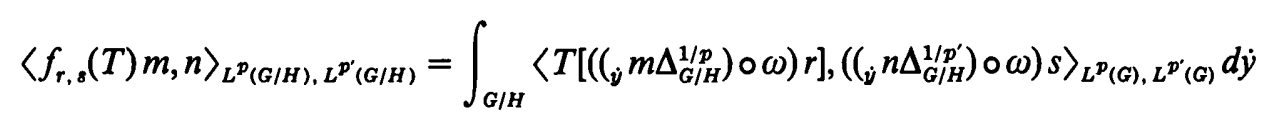

defines a bounded linear map $f_{r, 8}$ of $P M_{p}(G)$ into $P M_{p}(G / H)$. Conversely, for $k, l \in C_{00}(G)$,

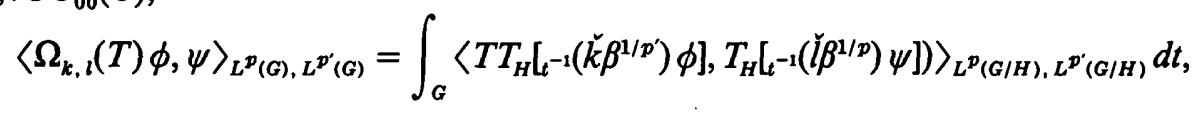

where $T_{H} \alpha(\dot{x})=\int_{H} \alpha(x h) d h$, defines a bounded linear map of $P M_{p}(G / H)$ into $P M_{p}(G)$. A compactness argument involving the maps $f_{r, s}$ allows one to define, in a canonical way, a linear contraction $R$ of $c v_{p}(G)$ (the norm closure in $P M_{p}(G)$ of $\left\{T \in P M_{p}(G) \mid \operatorname{supp} T\right.$ compact $\left.\}\right)$ onto $c v_{p}(G / H)$ which coincides on $L^{1}(G)$ with $T_{H}$. See [4] for details.

We also need several important subspaces of $P M_{p}(G): P F_{p}(G)$ is the norm closure of $L^{1}(G)$ in $P M_{p}(G)$ and $C_{p}(G)$ the set of all $T \in P M_{p}(G)$ such that $T S \in P F_{p}(G)$ for every $S \in P F_{p}(G)$. We recall that $P F_{p}(G) \subset c v_{p}(G) \subset C_{p}(G) \subset P M_{p}(G)$.

If we assume $G$ abelian and $p=2$, the preceding spaces have a very concrete and very simple description. Via the Fourier transform, $P F_{2}(G)$ is isomorphic to the space of all continuous functions on $\hat{G}$ vanishing at infinity, $c v_{2}(G)$ to the space of all bounded uniformly continuous functions on $\hat{G}$ and $C_{2}(G)$ to the space $C^{b}(\hat{G})$ of all bounded continuous functions on $\hat{G}$. Moreover, for $T \in C v_{2}(G), \widehat{R(T)}=\operatorname{Res}_{H^{+}} \hat{T}$ where $H^{\perp}=\{\chi \in \hat{G} \mid \chi(h)=1$ for every $h \in H\}$.

For $G$ arbitrary, $A_{2}(G)$ is the Fourier algebra of $G$ introduced and investigated by Eymard, $P F_{2}(G)$ is the reduced $C^{*}$-algebra of $G$ and $P M_{2}(G)$ is the von Neumann algebra of $G[6]$.

Let $W_{p}(G)$ be the norm dual of $P F_{p}(G)$; we identify it as a subalgebra of $C^{b}(G)$ in such a way that for all $f \in L^{1}(G)$ and $w \in W_{p}(G)$,

$$
\left\langle\lambda_{G}^{p}(f), w\right\rangle_{P F_{p}(G), w_{p}(G)}=\int_{G} f(x) \overline{w\left(x^{-1}\right)} d x .
$$

For $G$ abelian, $W_{2}(G)$ is isomorphic to the Banach space of all bounded Radon measures on $\hat{G}$. For $G$ amenable, $W_{2}(G)$ is the Fourier-Stieltjes algebra of $G$ [6]. In analogy with the abelian case, there is a duality between $C_{p}(G)$ and $W_{p}(G)$ : we can define it by $\langle T, w\rangle_{C_{p}(G), w_{p}(G)}=\lim \left\langle T \lambda_{G}^{p}\left(f_{i}\right), w\right\rangle_{P_{p}(G), w_{p}(G)}$ where $\left(f_{i}\right)_{i \in I}$ is any bounded approximate unit in $L^{1}(G)$ (see $\left.[2,3]\right)$. In [3], the interested reader can find, among other results, a detailed investigation of the duality between $C_{p}(G)$ and $W_{p}(G)$. We first need a little complement to that work.

LEMMA 1. Let $T \in c v_{p}(G), u, v \in W_{p}(G)$ and $w \in W_{p}(G / H)$. Then

(1) $\langle T, u v\rangle_{c_{p}(G), w_{p}(G)}=\langle u T, v\rangle_{c_{p}(G), w_{p}(G)}$

(2) $R(w \circ \omega T)=w R(T)$.

Proof. (1) Let $\left(u_{\alpha}\right)$ be a bounded approximate unit of $A_{p}(G)$, then we have by [2, Théorème 2, p. 136]

$$
\begin{aligned}
\langle T, u v\rangle_{C_{p}(G), w_{p}(G)} & =\lim \left\langle u_{\alpha} T, u v\right\rangle_{C_{p^{\prime}}(G), w_{p}(G)}=\lim \overline{\left\langle u_{\alpha} u v, T\right\rangle_{A_{p}(G), P M_{p}(G)}} \\
& =\lim \left\langle u_{\alpha} u T, v\right\rangle_{C_{p}(G), w_{p}(G)}=\langle u T, v\rangle_{C_{p}(G), w_{p}(G)} .
\end{aligned}
$$


(2) From [2, Théorème 3, p. 137], we have for all $v \in W_{p}(G / H)$

$$
\begin{aligned}
\langle R(w \circ \omega T), v\rangle_{C_{p}(G / H), w_{p}(G / H)} & =\langle w \circ \omega T, v \circ \omega\rangle_{C_{p}(G), w_{p}(G)}=\langle T, w \circ \omega v \circ \omega\rangle_{c_{p}(G), w_{p}(G)} \\
& =\langle R(T), w v\rangle_{C_{p}(G / H), w_{p}(G / H)}=\langle w R(T), v\rangle_{C_{p}(G / H), w_{p}(G / H)} .
\end{aligned}
$$

\section{A bijective correspondence between ideals of $A_{p}(G / H)$}

and invariant ideals of $A_{p}(G)$

Definitions. Let $I$ be a closed ideal of $A_{p}(G / H)$. The closure in $A_{p}(G)$ of the linear span of $\left\{u v \circ \omega \mid u \in A_{p}(G), v \in I\right\}$ is a closed ideal $e(I)$ of $A_{p}(G)$. Observe that $e(I)$ is invariant by right and left translations by elements of $H$. Let $I$ be closed ideal of $A_{p}(G)$. The closure in $A_{p}(G / H)$ of $T_{H}\left(I \cap C_{00}(G)\right)$ is a closed ideal $r(I)$ of $A_{p}(G / H)$.

In the $L^{1}$-case, for $G$ abelian, the corresponding definitions are the following ones. For a closed ideal $I$ of $L^{1}(H), e(I)$ is the closure in $L^{1}(G)$ of the linear span of $C_{00}(G) *_{H} I$. For a closed ideal $I$ of $L^{1}(G), r(I)$ is the closure in $L^{1}(H)$ of the linear span of $\left\{\operatorname{Res}_{H} f \mid f \in I * C_{00}(G)\right\}[11$, p. 168].

Let $I$ be closed ideal of $A_{p}(G)$, we recall that $I^{\perp}$ is the set of all $T \in P M_{p}(G)$ such that $\langle u, T\rangle_{A_{p}(G), P M_{p}(G)}=0$ for all $u \in I$. The amenability of $G$ implies that

$$
I^{\perp}=\left\{T \in P M_{p}(G) \mid u T=0 \text { for all } u \in I\right\} .
$$

The main result of this paragraph requires the following lemmas.

LEMMA 2. Let I be a closed ideal of $A_{p}(G)$ and $T \in P M_{p}(G)$ such that $R(u T) \in r(I)^{\perp}$ for every $u \in A_{p}(G)$. Then $T \in I^{\perp}$. $y \in G$,

Proof. Let $u \in I, v, w \in A_{p}(G) \cap C_{00}(G)$ and $r, s \in C_{00}(G)$. Observe that for every

$$
R\left(y_{y^{-1}} w{\overline{\tau_{p}}}^{P} *\left(\tau_{p} s\right)^{\nu} T\right)=f_{r, 8}\left(y^{-1} w T\right)
$$

(see [5, Proposition 3(iii), p. 98]). From [5, Proposition 13a), p. 104], we deduce that

$$
\int_{H} \overline{\check{v} * w\left(h^{-1}\right)} f_{r, s}\left(u_{h} T\right) d h=\int_{G} T_{H}\left(y^{-1} v u\right) f_{r, 8}\left(y^{-1} w T\right) d y=0 .
$$

This implies $f_{r, 8}(u T)=0$ and therefore $u T=0$.

LeMma 3. Let $I$ be a closed ideal in $A_{p}(G)$ such that $u_{h} \in I$ for $h \in H$ and $u \in I$. Then $R(v T) \in r(I)^{\perp}$ for every $v \in A_{p}(G)$ and $T \in I^{\perp}$.

Proof. It suffices to consider $v \in A_{p}(G) \cap C_{00}(G)$. Let $u \in I \cap C_{00}(G)$ and $r, s \in C_{00}(G)$. We have

$$
\int_{H} f_{r, 8}\left(v u_{h} T\right) d h=0
$$

From [5, Proposition 13b), p. 104], it follows that $T_{H}(u) f_{r, 8}(v T)=0$. This implies

$$
T_{H}(u) R\left(\tau_{p} r *\left(\tau_{p^{\prime}} s\right)^{\llcorner} v T\right)=0
$$

and therefore $T_{H}(u) R(v T)=0$. We finally obtain $R(v T) \in r(I)^{\perp}$.

LEMMA 4. Let I be a closed ideal of $A_{p}(G / H)$ and $T \in P M_{p}(G / H)$. Then $T \in I^{\perp}$ if and only if $\Omega_{k, l}(T) \in e(I)^{\perp}$ for every $k, l \in C_{00}(G)$. 
Proof. Recall that for $u \in A_{p}(G)$ and $v \in A_{p}(G / H)$, we have [5, Proposition 19, p. 111]

$$
u v \circ \omega \Omega_{k, l}(T)=u \Omega_{k, l}(v T) .
$$

THEOREM 5. Let $G$ be an amenable locally compact group and $H$ a closed normal subgroup of $G$.

(1) We have $I=r(e(I))$ for every closed ideal $I$ of $A_{p}(G / H)$.

(2) For every closed ideal I of $A_{p}(G)$ such that $u_{h} \in I$ for $u \in I$ and $h \in H$, we have also $I=e(r(I))$.

Hence $e$ is a bijection between the set of all closed ideals of $A_{p}(G / H)$ and the set of all closed ideals of $A_{p}(G)$ invariant under $H$.

Proof. (1) From the very definitions we get $r(e(I)) \subset I$. For the reverse inclusion, we shall show that $r(e(I))^{\perp} \subset I^{\perp}$. Let $T \in r(e(I))^{\perp}$, and consider $v \in r(e(I)), u \in A_{p}(G)$ and $k, l \in C_{00}(G)$. From $v R\left(u \Omega_{k, l}(T)\right)=R\left(u \Omega_{k, l}(v T)\right)$, we get $R\left(u \Omega_{k, l}(T)\right) \in r(e(I))^{\perp}$. Lemma 2 implies $\Omega_{k, l}(T) \in e(I)^{\perp}$, finally from Lemma 4 we conclude that $T \in I^{\perp}$.

(2) Taking into account (1) we need only to prove that for two closed ideals $I, J$ of $A_{p}(G)$ with $u_{h} \in I, v_{h} \in J$ for $h \in H, u \in I, v \in J$ and $r(I)=r(J)$, it follows that $I=J$. Let $T \in I^{\perp}$; by Lemma 3, $R(u T) \in r(J)^{\perp}$ for $u \in A_{p}(G)$. Lemma 2 implies $T \in J^{\perp}$.

Remarks. (1) For $A \subset G$, define $I(A)$ to be the ideal of $A_{p}(G)$ consisting of those functions which vanish on $A$. Let $F$ be a closed subset of $G / H$, then $e(I(F))=I\left(\omega^{-1}(F)\right)$ and $r\left(I\left(\omega^{-1}(F)\right)\right)=I(F)$.

(2) We conjecture that Theorem 5 is true for non-amenable groups. However amenability is used several times in our proof (existence of $R$, characterization of $\left.I^{\perp}, \ldots\right)$.

(3) Let $I$ be a closed ideal of $A_{p}(G)$ such that ${ }_{h} u \in I$ for every $u \in I$ and $h \in H$, then we have $u_{n} \in I$ for every $u \in I$ and $h \in H$. It suffices to apply the preceding theorem to the ideal $\{\tilde{u} \mid u \in I\}$ of $A_{p^{\prime}}(G)$.

It is possible to extend partially the second part of Theorem 5 to arbitrary closed ideals of $A_{p}(G)$.

Proposimion 6. Let I be a closed ideal of $A_{p}(G)$, then $e(r(I))$ coincides with $I_{H}$, the closure in $A_{p}(G)$ of the linear span of $\left\{u_{h} \mid u \in I, h \in H\right\}$. (Remark that $I_{H}$ is the smallest $H$-invariant closed ideal of $A_{p}(G)$ containing $H$.)

Proof. Because of Theorem 5, it suffices to verify that $r\left(I_{H}\right) \subset r(I)$, the converse inclusion being a direct consequence of $I \subset I_{H}$. Take $v \in I_{H} \cap C_{00}(G)$ and $\varepsilon>0$ and let $K$ be a compact neighbourhood of supp $v$. We recall the existence of $C>0$ such that $\left\|T_{H} u\right\|_{A_{p}(G / H)} \leqslant C\|u\|_{A_{p}(G)}$ for every $u \in A_{p}(G)$ with supp $u \subset K$ [14, lemme 1, p. 188]. Choose $w \in A_{p}(G)$ with $w=1$ on $\operatorname{supp} v$, supp $w \subset K$ and $\|w\|_{A_{p}(G)} \leqslant 2$. Now there exists $u_{1}, \ldots, u_{n} \in I$ and $h_{1}, \ldots, h_{n} \in H$ such that

Let $w_{j}=u_{j} w_{n_{j}^{-1}}$, so

$$
\left\|v-\sum_{i=1}^{n}\left(u_{j}\right)_{h_{s}}\right\|_{A_{p}(G)}<\frac{\varepsilon}{2 C} \text {. }
$$

$$
\left\|T_{H} v-\sum_{i=1}^{n} T_{H}\left(\left(w_{j}\right)_{h_{h}}\right)\right\|_{A_{\mathcal{p}}(G / H)}<\varepsilon
$$


From $T_{H}\left(\left(w_{j}\right)_{h_{j}}\right) \in r(I)$, we deduce finally $T_{H} v \in r(I)$ and the conclusion.

4. A natural inclusion of $\operatorname{Hom}_{A_{p^{(G / H)}}}\left(P M_{p}(G / H)\right)$ into $\operatorname{Hom}_{A_{p}(G)}\left(P M_{p}(G)\right)$

Definition. For $T \in c v_{p}(G)$ and $\phi \in c v_{p}(G)^{*}$, there is a unique $\phi \cdot T \in c v_{p}(G)$ with

$$
\phi(u T)=\overline{\langle u, \phi \cdot T\rangle_{A_{p}(G), P M_{p}(G)}} \text { for every } u \in A_{p}(G) .
$$

For $\phi, \psi \in c v_{p}(G)^{*}$, we define $(\phi \cdot \psi)(T)=\phi(\psi \cdot T)$ for $T \in c v_{p}(G)$.

With this operation $c v_{p}(G)^{*}$ becomes a Banach algebra. We also have

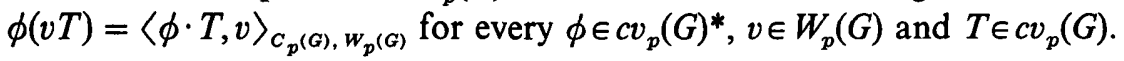

Proposition 7. For $\phi \in c v_{p}(G)^{*}$ and $T \in P M_{p}(G)$, there is a unique

such that

$$
\sigma_{G}(\phi)(T) \in P M_{p}(G)
$$

$$
\phi(u T)={\overline{\left\langle u, \sigma_{G}(\phi)(T)\right\rangle_{A_{p}(G), P M_{p}(G)}}}
$$

for every $u \in A_{p}(G) ; \sigma_{G}$ is a Banach algebra isometry from $c v_{p}(G)^{*}$ onto $\operatorname{Hom}_{A_{p}(G)}\left(P M_{p}(G)\right)$. For every $\Phi \in \operatorname{Hom}_{A_{p}(G)}\left(P M_{p}(G)\right)$ and $T \in c v_{p}(G)$, we have

$$
\sigma_{G}^{-1}(\Phi)(T)=\left\langle\Phi(T), 1_{G}\right\rangle_{C_{p^{(G)}}, w_{p^{(G)}}}
$$

REMARKS. (1) This proposition is not new! All these assertions can be deduced, for example, from [9, p. 140]. The fact that $\sigma_{G}$ is a bijective linear isometry is also due to Granirer [8, Proposition 2.1 , p. 160$]$. We present here a very short self-contained approach.

(2) $\sigma_{G}$ is onto if and only if $G$ is amenable [8, Theorem 2.1, p. 160].

(3) Note that $\sigma_{G}(\phi)(S)=\phi \cdot S$ for every $S \in c v_{p}(G)$.

Proof of Proposition 7. (I) $\sigma_{G}$ is a linear isometry.

It is clear that $\left\|\sigma_{G}(\phi)\right\| \leqslant\|\phi\|$. Let $\Phi \in \operatorname{Hom}_{A_{p}(G)}\left(P M_{p}(G)\right)$. Define $\phi \in c v_{p}(G)^{*}$ by $\phi(T)=\left\langle\Phi(T), 1_{G}\right\rangle_{c_{p^{(}(G)}, w_{p}(G)}$. We have $\|\phi\| \leqslant\|\Phi\|^{p}$ and, for $u \in A_{p}(G)$ and $T \in P M_{p}(G)$,

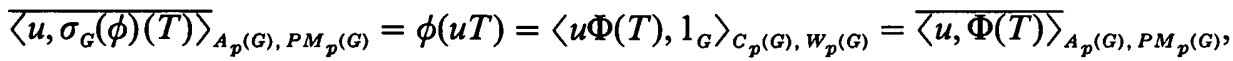
hence $\sigma_{G}(\phi)=\Phi$ and $\|\phi\|=\|\Phi\|$.

(II) $\sigma_{G}(\phi \cdot \psi)=\sigma_{G}(\phi) \sigma_{G}(\psi)$.

For $T \in P M_{p}(G)$ and $u \in A_{p}(G)$, we have

$$
\begin{aligned}
\left\langle u, \sigma_{G}(\phi) \sigma_{G}(\psi)(T)\right\rangle_{A_{p}(G), P M_{p}(G)} & =\phi\left(u\left(\sigma_{G}(\psi)(T)\right)\right)=\phi(\psi \cdot(u T)) \\
& =(\phi \cdot \psi)(u T)=\overline{\left\langle u, \sigma_{G}(\phi \cdot \psi)(T)\right\rangle_{A_{p}(G), P M_{p}(G)} .}
\end{aligned}
$$

THEOREM 8. Let $j$ be the map $\sigma_{G} \circ R^{*} \circ \sigma_{G / H}^{-1}$.

(1) $j$ is a Banach algebra isometry from $\operatorname{Hom}_{A_{p}(G / H)}\left(P M_{p}(G / H)\right)$ into $\mathrm{Hom}_{A_{p}(G)}\left(P M_{p}(G)\right)$. have

(2) For $T \in P M_{p}(G), u \in A_{p}(G), v \in W_{p}(G / H)$ and $\Phi \in \operatorname{Hom}_{A_{p}(G / H)}\left(P M_{p}(G / H)\right)$, we

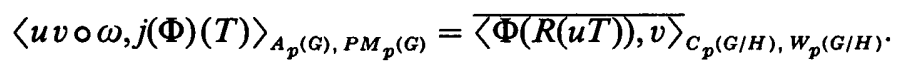

(3) Let I be a closed ideal of $A_{p}(G / H)$. Assume the existence of a projection $P$ of $P M_{p}(G / H)$ onto $I^{\perp}$ with $P \in \operatorname{Hom}_{A_{p}(G / H)}\left(P M_{p}(G / H)\right)$. Then $j(P)$ is a projection of $P M_{p}(G)$ onto $e(I)^{\perp}$. 
Proof. (1) From [4, Theorem 6, p. 21], we know that $R^{*}$ is an isometry from $c v_{p}(G / H)^{*}$ into $c v_{p}(G)^{*}$; so it suffices to show that $R^{*}$ is an algebra homomorphism. For $u \in A_{p}(G), T \in c v_{p}(G)$ and $\phi \in c v_{p}(G / H)^{*}$, we have

$$
\left\langle u, R\left(R^{*}(\phi) \cdot T\right)\right\rangle_{A_{p}(G / H), P M_{p}(G / H)}=\overline{\left\langle R^{*}(\phi) \cdot T, u \circ \omega\right\rangle_{c_{p}(G), w_{p}(G)}}
$$

[2, théorème 2, p. 136].

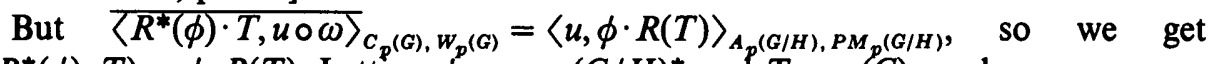
$R\left(R^{*}(\phi) \cdot T\right)=\phi \cdot R(T)$. Letting $\phi, \psi \in c v_{p}(G / H)^{*}$ and $T \in c v_{p}(G)$, we have

$\left(R^{*}(\phi) \cdot R^{*}(\psi)\right)(T)=\phi\left(R\left(R^{*}(\psi) \cdot T\right)\right)=\phi(\psi \cdot R(T))=\left(R^{*}(\phi \cdot \psi)\right)(T)$.

(2)

$$
\begin{aligned}
\langle v \circ \omega u, j(\Phi)(T)\rangle_{A_{p}(G), P M_{p}(G)} & =R^{*}\left(\sigma_{G / H}^{-1}(\Phi)\right)(v \circ \omega u T) \\
& =\left\langle\Phi(R(v \circ \omega u T)), 1_{G / H}\right\rangle_{C_{p}(G / H), w_{p}(G / H)} \\
& =\left\langle\Phi(v R(u T)), 1_{G / H}\right\rangle_{C_{p}(G / H), w_{p}(G / H)} \\
& =\langle\Phi(R(u T)), v\rangle_{C_{p}(G / H), w_{p}(G / H) .}
\end{aligned}
$$

(3) Letting $u \in A_{p}(G), v \in I$ and $T \in P M_{p}(G)$, we have

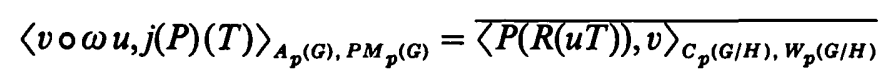

and therefore $j(P)(T) \in e(I)^{\perp}$. Let $T \in e(I)^{\perp}$. For $u \in A_{p}(G)$ and $v \in A_{p}(G / H)$, we have $R(u T) \in I^{\perp}$ and using (2)

$\langle v \circ \omega u, j(P)(T)\rangle_{A_{p^{\prime}(G), P M_{p}(G)}}=\langle v, R(u T)\rangle_{A_{p}(G / H), P M_{p}(G / H)}=\langle u v \circ \omega, T\rangle_{A_{p}(G), P M_{p}(G)} ;$ this implies $j(P)(T)=T$.

RemarK. Putting $v=1_{G / H}$ in (2), we get

$$
\langle u, j(\Phi)(T)\rangle_{A_{p}(G), P M_{p}(G)}=\left\langle\Phi(R(u T)), 1_{G / H}\right\rangle_{C_{p}(G / H), w_{p}(G / H)} .
$$

5. A conditional expectation of $\operatorname{Hom}_{A_{p}(G)}\left(P M_{p}(G)\right)$ onto $\operatorname{Hom}_{A_{p}(G / H)}\left(P M_{p}(G / H)\right)$.

$$
\text { An application }
$$

We first need some complements to [14, lemme 1] and [4, p. 13 and 17]. Then,

TheOREM 9. Let $u \in A_{p}(G), T \in P M_{p}(G / H), k, l \in C_{00}(G)$ and $k^{\prime}=\check{k} \beta^{1 / p^{\prime}}, l^{\prime}=\check{l} \beta^{1 / p}$.

$$
\left\|\Delta_{G / H}^{1 / p^{\prime}} T_{H}\left(u \tilde{k}^{\prime} * l^{\prime} \Delta_{G}^{-1 / p^{\prime}}\right)\right\|_{\left.A_{p^{(G / H}}\right)} \leqslant\|k\|_{p}\|l\|_{p^{\prime}}\|u\|_{A_{p}(G)}
$$

and

$$
\begin{gathered}
\left\langle u, \Omega_{k, l}(T)\right\rangle_{A_{p}(G), P M_{p}(G)}=\left\langle\Delta_{G / H}^{1 / p^{\prime}} T_{H}\left(\Delta_{G}^{-1 / p^{\prime}} u \tilde{k}^{\prime} * l^{\prime}\right), T\right\rangle_{A_{p}(G / H), P M_{p}(G / H)} . \\
R\left(u \Omega_{k, l}(T)\right)=\left(\Delta_{G / H}^{1 / p^{\prime}} T_{H}\left(\Delta_{G}^{-1 / p^{\prime}} \tilde{k}^{\prime} * l^{\prime} u\right)\right) T .
\end{gathered}
$$

Proof. (1) We only need to consider $u=\bar{r} * \check{s}$ with $r, s \in C_{00}(G)$. We have

where

$$
\left\langle\bar{r} * \check{S}, \Omega_{k, l}(T)\right\rangle_{A_{p}(G), P M_{p}(G)}=\int_{G}\langle w(t), T\rangle_{A_{p}(G / H), P M_{p}(G / H)} d t,
$$

$$
w(t)=\overline{\tau_{p}\left(T_{H}\left(t^{-1} k^{\prime} \tau_{p} r\right)\right)} *\left(\tau_{p^{\prime}}\left(T_{H}\left(c^{-1} l^{\prime} \tau_{p^{\prime}} s\right)\right)\right)^{r}
$$


It is easy to verify that $w$ is a continuous map from $G$ into the Banach space $A_{p}(G / H)$ and that

$$
\int_{G}\|w(t)\|_{A_{p^{(G / H)}}} d t \leqslant\|k\|_{p}\|l\|_{p^{\prime}}\|r\|_{p}\|s\|_{p^{\prime}}
$$

There is a unique $a \in A_{p}(G / H)$ with

$$
\langle a, S\rangle_{A_{p}(G / H), P M_{p}(G / H)}=\int_{G}\langle w(t), S\rangle_{A_{p}(G / H), P M_{p}(G / H)} d t
$$

for every $S \in P M_{p}(G / H)$. For $x \in G$ we obtain

$$
\begin{aligned}
a(\dot{x}) & =\int_{G}\left(\Delta_{G / H}^{1 / p^{\prime}} T_{H}\left(t^{-1} k^{\prime} \tau_{p}\right)^{*}\right) *\left(\Delta_{G / H}^{1 / p^{\prime}} T_{H}\left(t^{-1} l^{\prime} \tau_{p^{\prime}} s\right)\right)(\dot{x}) d t \\
& =\Delta_{G / H}^{1 / p^{\prime}}(\dot{x}) \int_{G} \int_{H} \int_{G} \Delta_{G}^{-1 / p^{\prime}}(x h) \overline{k^{\prime}\left(t^{-1} y^{-1}\right)} \overline{r(y)} l^{\prime}\left(t^{-1} y^{-1} x h\right) \check{s}\left(y^{-1} x h\right) d y d h d t \\
& =\Delta_{G / H}^{1 / p^{\prime}}(\dot{x}) T_{H}\left(\Delta_{G}^{-1 / p^{\prime}} \tilde{k}^{\prime} * l^{\prime} \tilde{r} * \tilde{S}\right)(\dot{x}),
\end{aligned}
$$

this implies $\left\langle\bar{r} * \check{S}, \Omega_{k, l}(T)\right\rangle_{A_{p}(G), P M_{p}(G)}=\langle a, T\rangle_{A_{p}(G / H), P M_{p}(G / H)}$ and

$$
\left\|\Delta_{G / H}^{1 / p^{\prime}} T_{H}\left(\Delta_{G}^{-1 / p^{\prime}} \tilde{k}^{\prime} * l^{\prime} \bar{r} * \mathfrak{s}\right)\right\|_{A_{p}(G / H)} \leqslant\|k\|_{p}\|l\|_{p^{\prime}}\|r\|_{p}\|s\|_{p^{\prime}} .
$$

(2) Using again [2, Théorème 2, p. 136], we have for $w \in W_{p}(G / H)$

$$
\begin{aligned}
& \left\langle R\left(u \Omega_{k, l}(T)\right), w\right\rangle_{C_{p}(G / H), w_{p}(G / H)}=\left\langle u \Omega_{k, l}(T), w \circ \omega\right\rangle_{C_{p}(G), w_{p}(G)} \\
& =\overline{\left\langle u w \circ \omega, \Omega_{k, 2}(T)\right\rangle_{A_{p}(G), P M_{p}(G)}}
\end{aligned}
$$

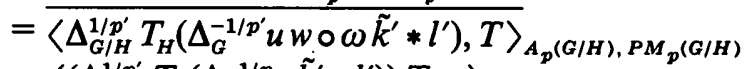

$$
\begin{aligned}
& =\left\langle\left(\Delta_{G / H}^{1 / p^{\prime}} T_{H}\left(\Delta_{G}^{-1 / p} u \tilde{k}^{\prime} * l^{\prime}\right)\right) T, w\right\rangle_{C_{p}(G / H), w_{p}(G / H)} .
\end{aligned}
$$

REMARK. The amenability of $G$ is not needed for (1).

THEOREM 10. There is a linear contraction $E$ of $\operatorname{Hom}_{A_{p}(G)}\left(P M_{p}(G)\right)$ onto $\operatorname{Hom}_{A_{p}(G / H)}\left(P M_{p}(G / H)\right)$ such that

(1) $E(j(\Psi))=\Psi$ for $\Psi \in \operatorname{Hom}_{A_{p}(G / H)}\left(P M_{p}(G / H)\right)$,

(2) $E(\Phi j(\Psi))=E(\Phi) \Psi$ for

$$
\Phi \in \operatorname{Hom}_{A_{p^{(G)}}}\left(P M_{p}(G)\right) \text { and } \Psi \in \operatorname{Hom}_{A_{p^{(G / H)}}}\left(P M_{p}(G / H)\right) \text {. }
$$

(3) Let $I$ be a closed ideal of $A_{p}(G / H)$. Assume the existence of a projection $P \in \operatorname{Hom}_{A^{(G)}}\left(P M_{p}(G)\right)$ of $P M_{p}(G)$ onto $e(I)^{\perp}$. Then $E(P)$ is a projection of $P M_{p}(G / H)$ onto $I^{\perp}$.

Proof. (I) Existence of $E$ with (1) and (2).

Let $X$ be the Banach space of all continuous bilinear functionals on $c v_{p}(G / H) \times c v_{p}(G)^{*}$. For a subset $C$ of $X, \bar{C}$ denotes the closure of $C$ in $X$ for the topology $\sigma\left(X, c v_{p}(G / H) \times c v_{p}(G)^{*}\right)$. For $k, l \in C_{00}(G / H)$, we define $\alpha_{k, l} \in X$ by $\alpha_{k, l}(T, \phi)=\phi\left(\Omega_{v_{p}(k), v_{p},(l)}(T)\right)$ where $v_{p}(k)=\check{\Delta}_{G}^{1 / p} \ddot{\beta}^{1 / p}\left(k \Delta_{G / H}^{1 / p}\right) \circ \omega$. For $\varepsilon>0$ and $F$ an arbitrary finite subset of $A_{p}(G / H)$, let $C_{F, e}$ be the set of all $\alpha_{k, l}$ such that $k, l \in C_{00}(G / H),\|k\|_{p}\|l\|_{p^{\prime}}=1$ and $\| u-u \bar{k} * \check{l}_{A_{p}(G / H)}<\varepsilon$ for every $u \in F$. There is $\alpha \in \cap\left\{\bar{C}_{F, \ell} \mid F\right.$ finite subset of $\left.A_{p}(G / H), \varepsilon>0\right\}$. For every $\phi \in c v_{p}(G)^{*}$ there is a unique $Q(\phi) \in c v_{p}(G / H)^{*}$ such that $\alpha(T, \phi)=Q(\phi)(T)$ for every $T \in c v_{p}(G / H)$. The map $Q$ is a linear contraction of $c v_{p}(G)^{*}$ into $c v_{p}(G / H)^{*}$. 
We show that $Q\left(R^{*}(\phi)\right)=\phi$ for every $\phi \in c v_{p}(G / H)^{*}$.

Choose $T \in c v_{p}(G / H)$ and $\varepsilon>0$. By the Cohen-Hewitt factorization theorem [13, 32.22], there is $w \in A_{p}(G / H)$ and $T^{\prime} \in c v_{p}(G / H)$ such that $T=w T^{\prime}$. There is also

$$
\alpha_{k, l} \in C_{\{w\}, \varepsilon / 2\left(1+\left\|T^{\prime}\right\|_{\mathcal{p}}\right)(1+\|\phi\|)} \text { such that }\left|\alpha\left(T, R^{*}(\phi)\right)-\alpha_{k, l}\left(T, R^{*}(\phi)\right)\right|<\frac{\varepsilon}{2} .
$$

We have $\alpha\left(T, R^{*}(\phi)\right)=Q\left(R^{*}(\phi)\right)(T), \alpha_{k, l}\left(T, R^{*}(\phi)\right)=\phi(\bar{k} * \zeta T)$ and

$$
|\phi(\bar{k} * \check{l} T)-\phi(T)|<\varepsilon / 2 \text {. }
$$

We conclude that $\left|Q\left(R^{*}(\phi)\right)(T)-\phi(T)\right|<\varepsilon$ and therefore $Q\left(R^{*}(\phi)\right)=\phi$.

For $\psi \in c v_{p}(G)^{*}$ and $\phi \in c v_{p}(G / H)^{*}$, we have $Q\left(\psi \cdot R^{*}(\phi)\right)=Q(\psi) \cdot \phi$.

Let $\varepsilon>0, S \in c v_{p}(G / H)$ and $w \in A_{p}(G / H)$. There is $\alpha_{k, l} \in C_{\{w\}, \varepsilon}$ with

$$
\left|\alpha\left(S, \psi \cdot R^{*}(\phi)\right)-\alpha_{k, l}\left(S, \psi \cdot R^{*}(\phi)\right)\right|<\frac{\varepsilon}{2} \quad \text { and }\left|\alpha(\phi \cdot S, \psi)-\alpha_{k, l}(\phi \cdot S, \psi)\right|<\frac{\varepsilon}{2} .
$$

We have $\alpha\left(S, \psi \cdot R^{*}(\phi)\right)=Q\left(\psi \cdot R^{*}(\phi)\right)(S), \alpha(\phi \cdot S, \psi)=(Q(\psi) \cdot \phi)(S)$ and

$$
\alpha_{k, l}\left(S, \psi \cdot R^{*}(\phi)\right)=\psi\left(R^{*}(\phi) \cdot \Omega_{v_{p}(k), v_{p}(l)}(S)\right) \text {. }
$$

For $u \in A_{p}(G)$ we have

where

$$
\begin{aligned}
\overline{\left\langle u, R^{*}(\phi) \cdot \Omega_{v_{p}(k), v_{p^{\prime}}(l)}(S)\right\rangle_{A_{p^{(}}(G), P M_{p}(G)}} & \left.=\phi\left(R\left(u \Omega_{v_{p^{\prime}}(k), v_{p^{\prime}}(l)}(S)\right)\right)\right) \\
& =\phi\left(\left(\Delta_{G / H}^{1 / p^{p}} T_{H}\left(\Delta_{G}^{-1 / p^{\prime}} u v\right)\right) S\right),
\end{aligned}
$$

We obtain

$$
v=\left(\left(v_{p}(k)\right)^{r} \beta^{1 / p}\right)^{\sim} *\left(v_{p^{\prime}}(l)\right)^{\sim} \beta^{1 / p} .
$$

$$
\left\langle u, R^{*}(\phi) \cdot \Omega_{v_{p}(k), v_{p}(l)}(S)\right\rangle_{A_{p}(G), P M_{p}(G)}=\left\langle u, \Omega_{v_{p}(k), v_{p}(l)}(\phi \cdot S)\right\rangle_{A_{p}(G), P M_{p}(G)},
$$

which implies

We finally get

$$
R^{*}(\phi) \cdot \Omega_{v_{p}(k), v_{p}(l)}(S)=\Omega_{v_{p}(k), v_{p}(l)}(\phi \cdot S) .
$$

$$
\left|Q\left(\psi \cdot R^{*}(\phi)\right)(S)-(Q(\psi) \cdot \phi)(S)\right|<\varepsilon .
$$

The map $E=\sigma_{G / H} \circ Q \circ \sigma_{G}^{-1}$ is therefore a linear contraction of $\operatorname{Hom}_{A_{p}(G)}\left(P M_{p}(G)\right)$ onto $\operatorname{Hom}_{A_{p}(G / H)}\left(P M_{p}(G / H)\right)$ which satisfies (1) and (2).

(II) Let $\Phi$ be an element of $\operatorname{Hom}_{A_{p}(G)}\left(P M_{p}(G)\right)$. For $T \in P M_{p}(G / H), F$ a finite subset of $A_{p}(G / H), \varepsilon, \varepsilon^{\prime}>0$, there is $\alpha_{k, l} \in C_{F, \varepsilon^{\prime}}$ with

$$
\left|\langle w, E(\Phi)(T)\rangle_{A_{p}(G / H), P M_{p}(G / H)}-\left\langle\Phi\left(\Omega_{v_{p}(k), v_{p}(l)}(T)\right), w \circ \omega\right\rangle_{C_{p}(G), w_{p}(G)}\right|<\varepsilon
$$

for every $w \in F$.

For there is $\alpha_{k, l} \in C_{F, \varepsilon^{\prime}}$ with $\left|\alpha\left(w T, \sigma_{G}^{-1}(\Phi)\right)-\alpha_{k, l}\left(w T, \sigma_{G}^{-1}(\Phi)\right)\right|<\varepsilon$ for every $w \in F$. We have

$$
\alpha\left(w T, \sigma_{G}^{-1}(\Phi)\right)=\left\langle\overline{\langle w, E(\Phi)(T)\rangle_{A_{p}(G / H), P M_{p}(G / H)}}\right.
$$

and

$$
\begin{aligned}
\alpha_{k, l}\left(w T, \sigma_{G}^{-1}(\Phi)\right) & =\left\langle\Phi\left(\Omega_{v_{p}(k), v_{p}(l)}(w T)\right), 1_{G}\right\rangle_{C_{p}(G), w_{p}(G)} \\
& =\left\langle w \circ \omega \Phi\left(\Omega_{v_{p}(k), v_{p},(l)}(T)\right), 1_{G}\right\rangle_{C_{p}(G), w_{p}(G)} \\
& =\left\langle\Phi\left(\Omega_{v_{p}(k), v_{p}(l)}(T)\right), w \circ \omega\right\rangle_{C_{p}(G), w_{p}(G)} .
\end{aligned}
$$

(III) We prove (3).

(a) For every $T \in P M_{p}(G / H)$ we have $E(P)(T) \in I^{\perp}$. 
Let $w$ be an element of the ideal $I$. For $\varepsilon>0$, there is $\alpha_{k, l} \in C_{\{w\}, \varepsilon}$ with

$$
\mid\langle w, E(P)(T)\rangle_{A_{p^{\prime}}(G / H), P M_{p^{(G / H)}}}-\left\langle\overline{\left\langle P \Omega_{v_{\left.p^{(}\right), v_{p^{\prime}}(l)}}(T), w \circ \omega\right\rangle_{C_{p^{\prime}}(G), w_{p}(G)}}\right|<\varepsilon .
$$

Choose $u \in A_{p}(G) \cap C_{00}(G)$ equal to 1 on a neighbourhood of the support of $P\left(\Omega_{v_{p}(k), v_{p^{\prime}}(l)}(T)\right)$. We have

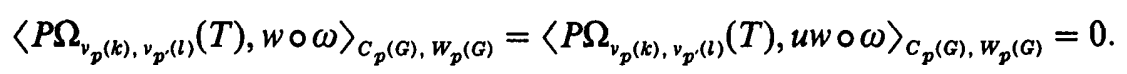

This implies $E(P)(T) \in I^{\perp}$.

(b) We prove that $E(P)(T)=T$ for every $T \in I^{\perp}$.

Let $\varepsilon>0$ and $w \in A_{p}(G / H)$. There is $\alpha_{k, l} \in C_{\{w\}, e / 2\left(1+\|T\|_{p}\right)}$ with

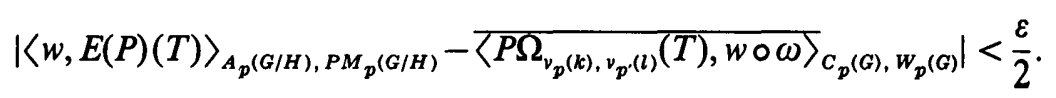

Taking into account Proposition 4 and Theorem 8, we obtain

This implies

$$
\begin{aligned}
\left\langle P \Omega_{v_{p}(k), v_{p^{\prime}}(l)}(T), w \circ \omega\right\rangle_{C_{\left.p^{(}\right), w_{p}(G)}} & =\left\langle\Omega_{v_{p^{\prime}}(k), v_{p^{\prime}}(l)}(T), w \circ \omega\right\rangle_{C_{p}(G), w_{p}(G)} \\
& =\left\langle R\left(\Omega_{v_{p}(k), v_{p^{\prime}}(l)}(T)\right), w\right\rangle_{C_{p}(G / H), w_{p}(G / H)} \\
& =\langle\bar{k} * \check{l} T, w\rangle_{C_{p}(G / H), w_{p}(G / H)} .
\end{aligned}
$$

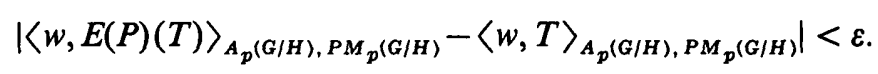

We conclude that $E(P)(T)=T$.

REMARK. We are not able to show that $E(j(\Psi) \Phi)=\Psi E(\Phi)$ for $\Psi \in \operatorname{Hom}_{A_{p}(G / H)}\left(P M_{p}(G / H)\right)$ and $\Phi \in \operatorname{Hom}_{A_{p}(G)}\left(P M_{p}(G)\right)$.

Proposition 11. Let $A$ be a commutative normed algebra with an approximate unit bounded by 1. Let I be a closed ideal of $A$ and $C>0$. The ideal I has an approximate unit bounded by $C$ if and only if there is a projection $P$ of $A^{*}$ onto $I^{\perp}$ with $\|\mathrm{id}-P\| \leqslant C$ and such that $P(a f)=a P(f)$ for $a \in A$ and $f \in A^{*}$.

This proposition is due to Françoise Lust-Piquard [15, pp. 7 and 15]. The condition on $C$ is not there but requires no new idea. See also [7, Proposition 6.4, p. 17].

COROLlaRY 12. Let I be a closed ideal of $A_{p}(G / H)$ and $C>0$. The ideal $I$ has an approximate unit bounded by $C$ if and only if $e(I)$ has an approximate unit bounded by $C$.

Proof. This corollary is a direct consequence of Proposition 11, Theorem 8(3) and Theorem 10(3).

REMARKS. (1) Assume that $I$ has an approximate unit bounded by $C$. It is possible to prove directly that $e(I)$ has an approximate unit bounded by $C$. It suffices to adapt the proof of the $L^{1}$-case (see [10, Lemma 1, p. 170; 1, Theorem, p. 392]).

Let $u \in e(I)$ and $\varepsilon>0$. There is $u_{1}, \ldots, u_{n} \in A_{p}(G)$ and $v_{1}, \ldots, v_{n} \in I$ such that

$$
\left\|u-\sum_{k=1}^{n} u_{k} v_{k} \circ \omega\right\|_{A_{p}(G)}<\frac{\varepsilon}{4(1+C)}
$$


There is $b \in A_{p}(G)$ such that $\|b\|_{A_{p}(G)} \leqslant 1$ and

$$
\left\|\sum_{k=1}^{n} u_{k} v_{k} \circ \omega-\left(\sum_{k=1}^{n} u_{k} v_{k} \circ \omega\right) b\right\|_{A_{p}(G)}<\frac{\varepsilon}{4(1+C)} .
$$

One can find $w \in I$ such that

$$
\|w\|_{A_{p}(G / H)} \leqslant C \text { and }\left\|v_{j}-v_{j} w\right\|_{A_{p}(G / H)}<\frac{3}{4\left(1+\sum_{k=1}^{n}\left\|u_{k}\right\|_{A_{p}(G)}\right)}
$$

for every $1 \leqslant j \leqslant n$. If we choose $d=b w \circ \omega$, we conclude that $d \in e(I),\|d\|_{A_{p}(G)} \leqslant C$ and $\|u-u d\|_{A_{p}(G)}<\varepsilon$.

The proof of the converse assertion seems to require the map $E$ (Theorem 10) and Proposition 11.

(2) As far as we know, Theorem 10 seems to be new even for $G$ abelian and $p=2$.

\section{References}

1. M. BekKa, 'On bounded approximate units in ideals of group algebras', Math. Ann. 266 (1984) 391-396.

2. J. Delaporte, 'Convoluteurs continus et topologie stricte', Harmonic Analysis. Proceedings, Luxembourg 1987. Lecture Notes in Mathematics, 1359 (Springer, Berlin, 1988), 135-141.

3. J. DeLAPORTE, 'Convoluteurs continus et topologie stricte', Thèse, Université de Lausanne 1989.

4. A. DerighetTI, 'A propos des convoluteurs d'un groupe quotient', Bull. Sci. Math. 107 (1983) 3-23.

5. A. Derighetri, 'Quelques observations concernant les ensembles de Ditkin d'un groupe localement compact', Monatsh. Math. 101 (1986) 95-113.

6. P. EYMARD, 'L'algèbre de Fourier d'un groupe localement compact', Bull. Soc. Math. France 92 (1964) $181-236$.

7. B. Forrest, 'Amenability and bounded approximate identities in ideals of $A(G)$ ', Illinois J. Math. 34 (1990) $1-25$.

8. E. E. Granirer, 'Some results on $A_{p}(G)$ submodules of $P M_{p}(G)$ ', Colloq. Math. 51 (1987) 155-163.

9. M. Grosser and V. LOSERT, 'The norm-strict bidual of a Banach algebra and the dual of $C_{u}(G)$ ', Manuscripta Math. 45 (1984) 127-146.

10. W. HAUENSCHILD and E. KANIUTH, 'Ideale mit beschränkter approximativer Eins in Gruppenalgebren von Moore-Gruppen', Manuscripta Math. 27 (1979) 167-182.

11. W. HAUENSCHILD and J. LUDWIG, 'The injection and the projection theorem for spectral sets', Monatsh. Math. 92 (1981) 167-177.

12. C. HERZ, 'Harmonic synthesis for subgroups', Ann. Inst. Fourier (Grenoble) (3) 23 (1973) 91-123.

13. E. HEWITt and K. A. Ross, Abstract harmonic analysis II (Springer, Berlin, 1970).

14. N. LoHOU', 'Remarques sur les ensembles de synthèse des algèbres de groupe localement compact', J. Functional Analysis 13 (1973) 185-194.

15. F. LUST-PIQUARD, 'Propriétés harmoniques et géométiques des sous-espaces invariants par translation de $L^{\infty}(G)$ ', Thèse, Université de Paris-Sud 1978.

16. H. REITER, Classical harmonic analysis and locally compact groups (Oxford University Press, London, 1968).

Institut de Mathématiques

Université de Lausanne

CH-1015 Lausanne

Switzerland 